\title{
Sloan iteration and Richardson extrapolation for Walsh series solutions of integral equations
}

\author{
W. F. Blyth* \\ V. Uljanov* \\ (Received 14 February 2005, revised 24 June 2005)
}

\begin{abstract}
Algorithms have been developed by the authors and co-workers for the solution of both Volterra and Fredholm integral equations by using the discontinuous wavelet packets known as the Walsh functions. These Walsh function methods are typically globally convergent of order one (and locally of order two).

The usual Walsh function method with $m=2^{n}$ terms approximates the solution with a piecewise constant function (constant on each sub-interval of width $1 / m$ ). For Fredholm integral equations, Sloan iteration of the Walsh series solution is globally convergent of order two.

For linear Fredholm integral equations of the second kind, we show in this paper that the Sloan iterates for Walsh series solution with
\end{abstract}

${ }^{*}$ Dept. Mathematics \& Statistics, RMIT University, Melbourne, Australia. mailto:bill.blyth@rmit.edu.au, vladimir.uljanov@rmit.edu.au

See http://anziamj.austms.org.au/V46/CTAC2004/Blyt for this article, (c) Austral. Mathematical Soc. 2005. Published July 26, 2005, amended August 2, 2005. ISSN 1446-8735 
$m$ and $2 m$ terms can be extrapolated by the Richardson method to give a function which approximates the solution with global fourth order convergence. This is very easy and efficient to implement. A second Richardson iteration for smooth problems results in sixth order convergence.

With Kulkarni's example (where the solution is only twice differentiable), a second Richardson iteration results in fifth order convergence. Our new method outperforms Kulkarni's method: it is easier implement, more accurate with 64 or more terms and the order of convergence is one higher.

As a further example, we use the nonlinear Chandrasekar integral equation for which a new solution method was recently proposed by the authors. Here, our numerical experiment shows that the Richardson extrapolation of the Sloan iteration functions is third order convergent.

The Walsh function methods are intuitively simple and robust. The straightforward implementation of Walsh series leads to schemes with low order convergence. However the implementation of the acceleration of convergence techniques described here is easy and efficient and provides schemes of higher order convergence.

\section{Contents}

1 Introduction

C692

2 Walsh functions

C694

3 The linear Fredholm equations and Walsh series solution C695

4 Solution using Sloan iteration and Richardson extrapolation

C697

5 Conclusion 


\section{Introduction}

New methods are always needed to solve integral equations because no single method works well for all such equations.

There has been considerable interest in solving differential and integral equations using techniques which involve the discontinuous wavelet packets known as the Walsh functions, see [2, 3, 4, 5, 6, 13] and references therein. One of the motivations for these developments is that these methods usually involve the use of the fast Walsh Fourier transform, which is faster than most corresponding transforms such as the trigonometric fast Fourier transform. Also, Walsh functions appear to be easily incorporated into a wide variety of robust general purpose algorithms.

In [3] an effective method to solve linear Volterra integral equations was introduced and it was shown how multigrid and Richardson extrapolation methods can be applied to improve efficiency. However, considerable preliminary work is needed before the Walsh series are introduced. The degenerate kernel approach requires a high computational time.

Subsequently [4], we showed that, by rewriting the problem in Fredholm form, the preliminary work and computational time (using the fast Walsh transform to obtain the double Walsh series for the modified kernel) to obtain the linear equation for the Walsh coefficients is greatly reduced. Volterra equations are solved effectively and efficiently with this new Walsh function method approach.

The straightforward implementation of Walsh function methods typically gives schemes that are globally convergent of order one (and locally of order two). This is why the idea of nested iteration using Richardson extrapolation 
of the Walsh series coefficients to seed each new level Picard iteration and to extrapolate to the final Walsh series result was introduced in [2] and further refined in $[3,4]$. This resulted in more efficient and more accurate solutions. However, as is usually the case, the Walsh function method with $m=2^{n}$ terms approximates the solution with a piecewise constant function (constant on each sub-interval of width $1 / m$ ).

In 1976, Sloan [12] proposed an iteration method to improve the convergence rates of Galerkin projection method solutions for Fredholm integral equations. Sloan's approach uses post-processing of the usual Galerkin solution. Recently, Kulkarni [10] proposed a new method which cannot be regarded as post-processing of Galerkin solutions. For a Galerkin method with first order convergence, Sloan iteration gives second order convergence and Sloan iteration of Kulkarni's method gives fourth order convergence.

For Fredholm integral equations, Sloan iteration of the Walsh series solution results in a continuous function which approximates the solution and is globally convergent of order two.

For linear Fredholm integral equations of second kind, we show that the Sloan iterates for Walsh series solution with $m$ and $2 m$ terms can be extrapolated by the Richardson method to give a function which approximates the solution with global fourth order convergence. This is easy and efficient to implement. Further extrapolation leads to still higher order convergence: a second Richardson iteration for smooth problems results in sixth order convergence. This is supported by the results of numerical experiment on test problems from Atkinson [1] and Kress [9].

In the case where the solution is less smooth, our numerical experiment with Kulkarni's example (where the solution is only twice differentiable) shows that the Richardson extrapolation remains globally convergent of fourth order. A second Richardson iteration in this case results in fifth order convergence. Our new method outperforms Kulkarni's method: it is more accurate with 64 or more terms and the order of convergence is one 
higher.

As a further example that is nonlinear, we use the Chandrasekar integral equation for which we recently proposed a new solution method [6]. Our numerical experiment (details omitted) shows that Richardson extrapolation of the Sloan iteration functions are convergent of order three.

The Walsh function methods are intuitively simple and robust. The straightforward implementation of Walsh series leads to schemes with low order convergence. However, the implementation of the acceleration of convergence techniques described here is easy and efficient (at almost no computational cost) and provide schemes of higher order convergence.

\section{Walsh functions}

The Walsh functions have many properties similar to those of the trigonometric functions. For example they form a complete, total collection of functions with respect to the space of square Lebesgue integrable functions. However, they are simpler in structure to the trigonometric functions because they take only the values 1 and -1 . They may be expressed as linear combinations of the Haar functions [8], so many proofs about the Haar functions carry over to the Walsh system easily. Moreover, the Walsh functions are Haar wavelet packets; see [14] for a good account of the properties of the Haar wavelets and other wavelets.

We use the ordering of the Walsh functions due to Paley [11]. Any function $f \in L^{2}[0,1)$ can be expanded as a series of Walsh functions

$$
f(x)=\sum_{i=0}^{\infty} c_{i} W_{i}(x) \quad \text { where } \quad c_{i}=\int_{0}^{1} f(x) W_{i}(x) d x .
$$

Fine [7] discovered an important property of the Walsh Fourier series: 
the $m=2^{n}$ th partial sum of the Walsh series of a function $f$ is piecewise constant, equal to the $L^{1}$ mean of $f$, on each subinterval $((i-1) / m, i / m)$. For this reason, Walsh series in applications are always truncated to $m=2^{n}$ terms. In this case, the coefficients $c_{i}$ of the Walsh (-Fourier) series are

$$
c_{i}=\sum_{j=0}^{m-1} \frac{1}{m} W_{i j} f_{j},
$$

where $f_{j}$ is the average value of the function $f(x)$ in the $j$ th interval of width $1 / m$ in the interval $(0,1)$, and $W_{i j}$ is the value of the $i$ th Walsh function in the $j$ th subinterval. The order $m$ Walsh matrix, $\mathcal{W}_{m}$, has elements $W_{i j}$.

\section{The linear Fredholm equations and Walsh series solution}

We consider Fredholm integral equations of the second kind of the form

$$
y(x)=g(x)+\int_{0}^{1} K(x, t) y(t) d t .
$$

Look at three examples.

1. The Kress test problem with solution $y(x)=e^{-x}$ is taken from $[9$, p.158]:

$$
y(x)=e^{-x}-\frac{1}{2}+\frac{e^{-x-1}}{2}+\int_{0}^{1} \frac{x+1}{2} e^{-x t} y(t) d t
$$

2. The Atkinson test problem with solution $y(x)=e^{x}$ istaken from $[1$, p.102]:

$$
y(x)=e^{x}+\frac{1}{2}\left(\frac{1-e^{x+1}}{x+1}\right)+\frac{1}{2} \int_{0}^{1} e^{x t} y(t) d t .
$$


3. The Kulkarni test problem with solution unknown, although $y \in$ $C^{2}[0,1]$, is taken from [10]:

$$
y(x)=1+x^{5 / 2}+\int_{0}^{1} \frac{1}{1+(x-t)^{2}} y(t) d t .
$$

To solve a linear Fredholm integral equation [5], we represent functions $y$ and $g$ by their Walsh series, truncated to $m$ terms. The kernel $K(x, t)$ is approximated by a truncated double Walsh series

$$
K(x, t)=\sum_{i=0}^{m-1} \sum_{j=0}^{m-1} k_{i j} W_{i}(x) W_{j}(t),
$$

where

$$
k_{i j}=\int_{0}^{1} \int_{0}^{1} K(x, t) W_{i}(x) W_{j}(t) d x d t
$$

and it is easy to show that the integral equation can be rewritten as the simple linear problem

$$
\mathbf{c}_{m}=\mathbf{g}_{m}+K_{m} \mathbf{c}_{m}
$$

where $K_{m}$ is the matrix with elements $k_{i j}$. This can be solved directly for simple problems and low $m$, but would usually be solved by ordinary (Picard) iterations.

The first task is to calculate the matrix containing the coefficients of double Walsh series $K_{m}=\left(k_{i j}\right)$. If $m=2^{n}$ (as is always the case in applications), then $K_{m}=\frac{1}{m^{2}} \mathcal{W}_{m} \bar{K}_{m} \mathcal{W}_{m}$. This is the double Walsh transform of the matrix $\bar{K}_{m}$ of the average values of $K(x, t)$ on all subintervals. Since the area of each of these $2 \mathrm{D}$ subintervals is $1 / \mathrm{m}^{2}$, we rewrite this as

$$
K_{m}=\mathcal{W}_{m} A_{m} \mathcal{W}_{m}
$$

where $A_{m}$ is the matrix of the double integrals of $K(x, t)$ over the subintervals. 
For the $i$ th $x$-subinterval and $j$ th $t$-subinterval of a square subregion, the element of $A_{m}$ is

$$
a_{i j}=\int_{(i-1) / m}^{i / m} \int_{(j-1) / m}^{j / m} K(x, t) d t d x .
$$

\section{Solution using Sloan iteration and Richardson extrapolation}

The Sloan iterate, $\tilde{y}_{m}$, is defined in the usual way as

$$
\tilde{y}_{m}(x)=g(x)+\int_{0}^{1} K(x, t) y_{m}(t) d t .
$$

However, the $y_{m}(t)$ in the integrand is the Walsh series truncated to $m=2^{n}$ terms: the approximate solution of equation (3). Thus $y_{m}(t)$ is piecewise constant (equal to $\bar{y}_{i}$, the average value of $y$ on the $i$ th subinterval of width $1 / m$ ) and the integration is particularly simple: it is a sum of integrals

$$
\int_{0}^{1} K(x, t) y_{m}(t) d t=\sum_{i=0}^{m-1} \bar{y}_{i} \int_{i / m}^{(i+1) / m} K(x, t) d t .
$$

For the Atkinson example 2, equation (5),

$$
\int_{i / m}^{(i+1) / m} K(x, t) d t=\frac{e^{(i+1) / m}-e^{i / m}}{2 x},
$$

and the Sloan iterate for $m=4$ is the continuous function

$$
\begin{aligned}
\tilde{y}_{4}= & \frac{1}{x(x+1)}[-0.5647833767062312-0.06478337670623124 x \\
& +e^{x / 4}(-0.16010441943408682-0.16010441943408682 x)
\end{aligned}
$$




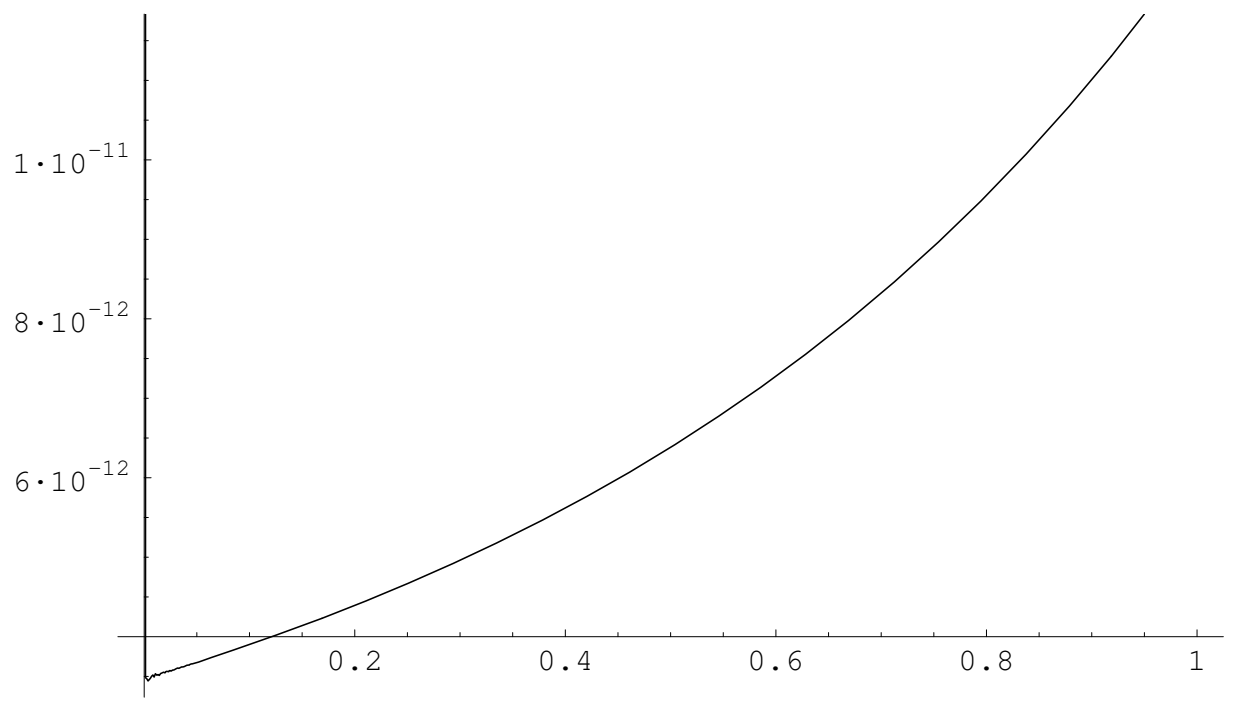

FiguRE 1: The error function for the Richardson Richardson extrapolation of the Sloan iterate, $\mathrm{yRiRi}_{m}$ with $m=64$, for the Atkinson problem 2.

$$
\begin{aligned}
& +e^{x / 2}(-0.20558795583134673-0.20558795583134673 x) \\
& +e^{(3 x) / 4}(-0.2639921252256509-0.2639921252256509 x) \\
& \left.+e^{x}\left(1.1944678771973156+0.8353269629677931 x+x^{2}\right)\right]
\end{aligned}
$$

Since these Sloan iterates have global convergence of order two, we introduce a Richardson extrapolation of the Sloan iteration functions to give a Richardson extrapolation function

$$
\mathrm{yRi}_{2 m}(x)=\frac{4}{3} \tilde{y}_{2 m}-\frac{1}{3} \tilde{y}_{m} .
$$

Our numerical experiments show that this Richardson extrapolation for linear problems is convergent of order four. Thus we use Richardson extrapolation 
TABLE 1: $L^{\infty}$ errors and the error ratios using Sloan iteration, a Richardson extrapolation and two Richardson extrapolations for the Atkinson problem 2.

\begin{tabular}{|r|ll|ll|ll|}
\hline$m$ & Sloan & $r$ & Rich & $r$ & RichRich & $r$ \\
\hline 4 & $1.86 \times 10^{-2}$ & & & & \\
8 & $4.68 \times 10^{-3}$ & 3.961 & $6.05 \times 10^{-5}$ & & & \\
16 & $1.17 \times 10^{-3}$ & 3.990 & $3.83 \times 10^{-6}$ & 15.80 & $5.12 \times 10^{-8}$ & \\
32 & $2.94 \times 10^{-4}$ & 3.998 & $2.40 \times 10^{-7}$ & 15.95 & $8.12 \times 10^{-10}$ & 63.1 \\
64 & $7.34 \times 10^{-5}$ & 3.999 & $1.50 \times 10^{-8}$ & 15.99 & $1.27 \times 10^{-11}$ & 63.8 \\
128 & $1.84 \times 10^{-5}$ & 4.000 & $9.37 \times 10^{-10}$ & 16.00 & $2.02 \times 10^{-13}$ & 62.9 \\
256 & $4.59 \times 10^{-6}$ & 4.000 & $5.87 \times 10^{-11}$ & 16.00 & $6.80 \times 10^{-15}$ & 29.7 \\
\hline
\end{tabular}

a second time to give the smooth solution function

$$
\mathrm{yRiRi}_{2 m}(x)=\frac{16}{15} \mathrm{yRi}_{2 m}-\frac{1}{15} \mathrm{yRi}_{m} .
$$

These Richardson extrapolations are obtained at almost no cost, but give schemes that are convergent of order six for smooth problems.

The error for the double Richardson extrapolation of the Sloan iteration for the Atkinson problem 2 is plotted in Figure 1. This is for the $m=64$ case (which requires the usual Walsh series solution and Sloan iteration for $m=16,32$ and 64$)$. The exact solution is $e^{x}$ and the maximum error is at $x=1$. In Table 1 we use $L^{\infty}$ errors. It is clear that the sequence of the $y \mathrm{RiRi}_{m}$ is sixth order convergent and the limit of precision has been reached for $y \mathrm{RiRi}_{256}$. Similarly for the Kress problem 1 which also exhibits sixth order convergence for the $y \mathrm{RiRi}_{m}$ and precision limits are evident in $\mathrm{yRiRi}_{128}$, see Table 2.

The Kulkani problem has a solution that is unknown, but is $C^{2}$. Precision limits were reached with yRiRi $i_{256}$ and so yRiRi 128 was used to approximate the exact solution. Errors were calculated at one of the points used in [10]. Errors at the other point used by Kulkarni were quite similar and slightly higher (by $<50 \%$ ). For this less smooth example the $\mathrm{yRiRi}_{m}$ is fifth order 
TABLE 2: Errors at $x=255 / 512$ and the error ratios using Sloan iteration, a Richardson extrapolation and two Richardson extrapolations for the Kress problem 1.

\begin{tabular}{|r|ll|ll|ll|}
\hline$m$ & Sloan & $r$ & Rich & $r$ & RichRich & $r$ \\
\hline 4 & $2.34 \times 10^{-3}$ & & & & & \\
8 & $5.86 \times 10^{-4}$ & 3.9993 & $1.33 \times 10^{-7}$ & & & \\
16 & $1.46 \times 10^{-4}$ & 3.9998 & $8.69 \times 10^{-9}$ & 15.29 & $4.13 \times 10^{-10}$ & \\
32 & $3.66 \times 10^{-5}$ & 4.0000 & $5.49 \times 10^{-10}$ & 15.82 & $6.48 \times 10^{-12}$ & 63.6 \\
64 & $9.15 \times 10^{-6}$ & 4.0000 & $3.44 \times 10^{-11}$ & 15.95 & $1.14 \times 10^{-13}$ & 56.6 \\
128 & $2.29 \times 10^{-6}$ & 4.0000 & $2.17 \times 10^{-12}$ & 15.90 & $1.47 \times 10^{-14}$ & 7.81 \\
256 & $5.72 \times 10^{-7}$ & 4.0000 & $1.48 \times 10^{-13}$ & 14.66 & $1.33 \times 10^{-14}$ & 1.10 \\
\hline
\end{tabular}

TABLE 3: Errors at $x=1 / 512$ and the error ratios using Sloan iteration, a Richardson extrapolation and two Richardson extrapolations for the Kulkarni problem 3.

\begin{tabular}{|r|ll|ll|ll|}
\hline$m$ & Sloan & $r$ & Rich & $r$ & RichRich & $r$ \\
\hline 4 & $2.72 \times 10^{-2}$ & & & & & \\
8 & $7.04 \times 10^{-3}$ & 3.870 & $3.07 \times 10^{-4}$ & & & \\
16 & $1.77 \times 10^{-3}$ & 3.967 & $1.93 \times 10^{-5}$ & 15.90 & $1.27 \times 10^{-7}$ & \\
32 & $4.43 \times 10^{-4}$ & 3.992 & $1.21 \times 10^{-6}$ & 15.95 & $3.57 \times 10^{-9}$ & 35.6 \\
64 & $1.09 \times 10^{-4}$ & 3.998 & $7.62 \times 10^{-8}$ & 15.90 & $1.11 \times 10^{-10}$ & 32.1 \\
\hline
\end{tabular}

convergent, see Table 3.

\section{Conclusion}

The direct solution of Fredholm integral equations using Walsh function methods results in an approximate solution which is a truncated Walsh series: a piecewise constant function. Straightforward implementation of these Walsh function methods is only globally convergent of order one. Our 
Richardson seeded nested iteration method, with extrapolation [2, 3, 4], is superior: it is efficient and it reduces the total error. However, the solution is still a Walsh series.

A Sloan iteration of a straightforward Walsh function method solution results in a continuous function and has global convergence of order two. For linear Fredholm integral of second kind, we show in this paper that the simple idea of post processing the Walsh series solution using a Sloan iteration followed by Richardson extrapolation of the Sloan iteration function gives easily implemented, efficient and high order convergent schemes. This solution is a function (not nodal values) and so there is no need to interpolate to approximate the solution at other than nodal points!

The approach introduced here can be extended to nonlinear problems. As an example that is nonlinear, the Chandrasekar integral equation has a new solution method recently proposed by the authors [6]. Error estimates were derived for our Walsh function method solution of the Chadrasekhar equation. The method was shown to be globally convergent of order one, with the Sloan iteration globally convergent of order two, so the method of this paper can be utilized. Here, numerical experiment (details omitted) shows that the Richardson extrapolation of the Sloan iteration functions are convergent of order three.

In further work, we will undertake the analysis required to derive error estimates for this new, accurate and efficient approach to the solution of Fredholm integral equations.

Acknowledgment: we thank Bob Anderssen for drawing our attention to the paper by Kulkarni. 


\section{References}

[1] K. E. Atkinson. The Numerical Solution of Integral Equations of the Second Kind. Cambridge University Press, 1997. C693, C695

[2] W. F. Blyth, R. L. May and P. Widyaningsih. The Solution of Integral Equations Using Walsh Functions and a Multigrid Approach. In J. Noye et al., editors, Computational Techniques and Applications: CTAC97, Proceedings, 99-106. World Scientific, Singapore, 1998. C692, C693, C701

[3] W. F. Blyth, R. L. May and P. Widyaningsih. Solution of Separable Volterra Integral Equations Using Walsh Functions and a Multigrid Approach. In M. Pemberton, I. Turner and P. Jacobs, editors, Engineering Mathematics and Applications Conference: EMAC 2002 Proceedings, 85-90. The Inst. of Engineers, Australia, 2002. C692, C693, C701

[4] W. F. Blyth, R. L. May and P. Widyaningsih. Volterra Integral Equations solved in Fredholm form using Walsh functions. ANZIAM J., 45(E), ppC269-C282, 2004. http://anziamj. austms.org.au/V45/CTAC2003/Blyt C692, C693, C701

[5] W. F. Blyth and V. Uljanov. Numerical Solution of Weakly Singular Fredholm Integral Equations using Walsh Functions. In R. L. May and A. K. Easton, editors, Computational Techniques and Applications: CTAC-95, Proceedings, 137-143. World Scientific, Singapore, 1996. C692, C696

[6] W. F. Blyth and V. Uljanov. Numerical Solution of the Chandrasekar Nonlinear Fredholm Integral Equation using Walsh Functions. in preparation. C692, C694, C701 
[7] N. J. Fine. On the Walsh Functions. Trans. Amer. Math. Soc., 65, 372-414, 1949. C694

[8] A. Haar. Zur Theorie der orthogonalen Funktionen systeme. Math. Ann., 69, 331-371, 1910. C694

[9] R. Kress. Linear Integral Equations. Springer-Verlag, 1989. C693, C695

[10] R. Kulkarni. A superconvergence result for solutions of compact operator equations. Bull. Austral. Math. Soc., 68, 517-528, 2003. C693, C696, C699

[11] R. E. A. C. Paley. A remarkable series of orthogonal functions (I \& II). London Math. Soc., 34, 241-264 \& 265-279, 1932. C694

[12] I. H. Sloan. Improvement by Iteration for Compact Operator Equations. Math. Comp. 30, 758-764, 1976. C693

[13] B. G. Sloss and W. F. Blyth. A Walsh function method for a nonlinear Volterra integral equation. J. Franklin Inst., 340, 25-41, 2003. C692

[14] P. Wojtaszczyk. A mathematical introduction to wavelets. Cambridge University Press, 1997. C694 